\title{
Évolution des effets de sens de la construction aller + infinitif
}

\author{
Milena Anđelić
}

Faculté de Philologie, Université de Belgrade*

Dans cet article, nous essaierons de vérifier le bien-fondé des arguments de Vet et de Bres sur l'évolution du sens de la construction aller+infinitif, qui aurait eu une lecture purement aspectuelle dans la langue française d'autrefois. Pour vérifier cette hypothèse, nous analyserons les exemples tirés des textes du XII ${ }^{\mathrm{e}}$ au XVI siècles et nous déterminerons s'ils s'intègrent dans un des trois niveaux d'interprétation de cette construction : 1) le sens spatial ; 2) l'aspect prospectif ; 3) le futur périphrastique. Dans la langue française préclassique on devrait retrouver des exemples qui suivent les deux premiers niveaux d'interprétation, mais pas encore le troisième, qui n'émergerait complètement que vers le $\mathrm{XVII}^{\mathrm{e}}$ siècle.

Mots-clés : l'aspect prospectif, la phrase préparatoire, le futur périphrastique, la grammaticalisation, le temps verbal.

\section{Introduction}

Dans un court commentaire sur la diachronie de la construction aller + infinitif, Co Vet affirme que « les formes [de la construction aller + inf.] qui avaient d'abord un sens aspectuel seulement en sont venues à pouvoir fonctionner comme un temps » (Vet 2011 : 24). De même, Bres et Labeau en donnent un cadre temporel : « [le sens aspectuel] se développe entre la fin du XIII ${ }^{\mathrm{e}}$ et le XVI ${ }^{\mathrm{e}}$ siècle » (Bres \& Labeau 2013 : 19).

En nous inspirant de ces informations sur l'évolution de la construction aller + inf., nous nous proposons d'observer son fonctionnement dans les exemples tirés des textes datant $\mathrm{du} \mathrm{XII}^{\mathrm{e}}$ au XVI ${ }^{\mathrm{e}}$ siècles, afin de vérifier en pratique les affirmations des linguistes.

Nous définirons d'abord les trois niveaux d'interprétation de la construction aller + infinitif, qui existent tous en français contemporain : 1. le sens spatial, 2. l'aspect prospectif, et 3. le futur périphrastique. Ensuite, à partir de ce système

\footnotetext{
*milenandelic@hotmail.com
} 
à trois niveaux, nous procéderons à l'analyse des exemples. Les corpus de textes utilisés sont la Base de français médiéval (BFM) pour les textes du XII ${ }^{\mathrm{e}}$ au $\mathrm{Xv}^{\mathrm{e}}$ siècle et Frantext pour les exemples du Xvi ${ }^{\mathrm{e}}$ siècle.

\section{Niveaux d'interprétation}

En français (moderne), il est possible de distinguer trois interprétations possibles pour la construction aller + infinitif: le sens spatial, l'aspect prospectif et le futur périphrastique.

\subsection{Le sens spatial}

L'effet de sens spatial serait à l'origine des autres emplois de la construction aller + infinitif (Rey 2016 ; Bres \& Labeau 2013), étant attesté en même temps que la construction elle-même, au XI ${ }^{\mathrm{e}}$ siècle. Puisque les deux verbes gardent leur sémantisme d'origine, on constate que la construction n'est pas encore grammaticalisée. De même, nous ne pouvons pas encore la traiter comme une périphrase, puisqu'elle exprime non pas un seul, mais bien deux signifiés - celui du verbe aller et celui du verbe à l'infinitif.

C'est le verbe aller qui dénote le mouvement nécessaire pour que l'action du verbe à l'infinitif soit réalisée. Le sujet se déplace (aller) en vue d'accomplir une seconde action (infinitif). Nous pouvons nous poser la question si le laps de temps implicite entre l'action de déplacement et l'action intentionnée est à l'origine de l'interprétation prospective.

L'effet de sens spatial permet au verbe conjugué de prendre n'importe quelle forme temporelle, alors que ce ne sera pas le cas pour les niveaux d'interprétation prospectif et futural.

\subsection{L'aspect prospectif}

La construction aller + infinitif, en tant que périphrase aspectuelle, décrit la phase préparatoire de l'action du verbe à l'infinitif. Le verbe aller devient un (semi-)auxiliaire et cesse de dénoter le mouvement d'un point à $\mathrm{A}$ un point $\mathrm{B}$ afin de faciliter la réalisation de l'action suivante. Le verbe à l'infinitif est désormais traité comme « verbe principal ».

À l'opinion de Vet (Vet 2008 : 21), l'interprétation prospective est l'interprétation par défaut en français contemporain. Pour pouvoir identifier une construction comme étant prospective, il est nécessaire d'avoir un nombre d'indices dans le contexte précédent, qui constitueraient un point d'appui. Ces indices peuvent être de natures différentes : « la décision du locuteur de faire l'action 
future, son pressentiment, sa conscience qu'un événement nouveau de manquera pas de se produire » (Stanojević 2014: 197). À cause de la présence des indices, les locuteurs ont l'impression de proximité temporelle par rapport au moment de la parole.

Contrairement à l'interprétation spatiale, l'auxiliaire aller peut seulement prendre la forme d'indicatif présent, ou d'indicatif imparfait si le point de référence R est situé dans le passé (Bres \& Labeau 2013 : 20). Les autres formes temporelles de l'auxiliaire excluent l'interprétation prospective parce qu'elles paraissent rétablir le sémantisme du verbe.

Le Dictionnaire du Moyen Français (DMF) donne quelques explications dans l'article du verbe aller qui concernent l'interprétation d'aller + infinitif dans les textes anciens :

si aller est au présent [dans un contexte au passé (simple)], la construction est l'équivalent d'un passé simple ; c'est la forme fréquente pour introduire le discours indirect et lui va dire...;

si aller est au présent [dans un contexte au présent (historique)], la construction est l'équivalent d'un présent (historique);

si aller est à l'imparfait, il s'agit d'une périphrase [prospective] d'action imminente ;

si aller est au passé simple, il s'agit s'une périphrase inchoative, marquant la soudaineté.

Nous verrons, à partir des exemples, qu'en français moyen et préclassique, il est possible de confondre les interprétations spatiale et prospective, parce qu'il est difficile de décider définitivement quel effet de sens est présent. Peut-être estce aussi parce que les deux interprétations ne s'excluent pas complètement.

\subsection{Le futur périphrastique (le futur proche)}

À la différence des cas où il s'agit de périphrase aspectuelle, la périphrase aller + infinitif à l'effet de sens futural est remplaçable par le futur simple (FS) dans les contextes futuraux, souvent en présence d'un adverbial temporel. De là provient la problématique dans l'analyse des exemples de futur périphrastique (FP) en français moderne. En effet, dans les exemples où il est impossible d'utiliser un FS à la place d'un FP, il faudrait envisager une autre lecture de la construction. En général, les interprétations spatiale et futurale ne prêtent pas à confusion, alors qu'il est souvent important de faire une analyse plus poussée du contexte afin de décider si ladite construction est futurale ou prospective.

L'une des explications possibles de cet état de choses serait que le FP a évolué à partir de la lecture aspectuelle, sans pourtant la remplacer. Néanmoins, au lieu de créer seulement l'impression d'une réalisation imminente, la distance 
temporelle du FP entre le moment d'énonciation (S) et le point de référence (R) est variable - de quelques secondes à plusieurs années. En outre, un FP se passe facilement d'indices nécessaires à une lecture aspectuelle.

D'après le DMF, « à la première personne, aller + inf. semble avoir déjà la valeur de futur proche qu'il a en français moderne » même dans un texte en moyen français. Puisque dans notre article nous allons traiter seulement un nombre d'exemples restreint à travers une époque où le FP est beaucoup plus rare que les autres lectures, nous ne pourrons que brièvement aborder cette problématique.

\section{Aller + infinitif dans les textes du XII au XVI siècle: analyse des exemples}

Dans cette partie, nous analyserons quelques exemples pour tenter de montrer comment la construction aller + infinitif était utilisée en moyen français et en français préclassique. Nous avons consulté le corpus BFM pour les textes du $\mathrm{XII}^{\mathrm{e}}$ au XV ${ }^{\mathrm{e}}$ siècle, et le corpus Frantext pour les textes du XVI ${ }^{\mathrm{e}}$ siècle. Nous présenterons ici seulement les exemples où le verbe aller est soit au présent soit à l'imparfait de l'indicatif, parce que ce sont les cas où différentes interprétations peuvent s'appliquer.

Dans les exemples suivants, où la construction a une valeur prospective, le verbe aller est au présent, mais dans un contexte au passé, ce qui élimine l'interprétation futurale. Le verbe à l'infinitif est un verbe déclaratif ( $c f$. DMF) introduisant le discours indirect : il ne s'agit pas seulement du verbe dire, on rencontre également appeler, (ra)conter, répondre.

1) Dreit a Orenge les paiens de la terreVont chasçant le bon marchis Willame. Vint a la porte, mais nel trovat mie overte.

Serrement le porter en va apeler :

« Ohi, porter frere, lai mei lainz entrer!» (ChGui, v. 2212-2216)

2) et vint a la tente des deux freres qui le bienviengnerent moult. Et il leur va accointier comment le roy estoit fort blecié, et comment il leur prioit humblement qu'il leur pleust a venir devers lui pour eulx mercier (Mél : 115)

3) Si se bessa de rechef et redressa le roy d'Espaigne, lequel va commancer a dire en se desconfortant et criant a haulte voix : " Trescrestien, hault, et puissant roy, je vous mercie humblement $[\ldots] »($ Rom : 4)

4) Le roy d'Angleterre pour ces parolles ce print moult fort a rire et luy va respondre : «Par Dieu, mon amy, il fauldroit avoir des holiffans grant planté a porter tant de maisons. » (Rom : 39)

5) Quant les chariotz et les deux cens hommes d'armes eurent passé, le roy $v a$ dire que l'on allast disner ce pendant (Rom : 58) 
6) « Je te prie, mon amy [...] compte-moy la sorte de ton entreprinse [...].» Le gentil homme luy vat compter comme elle avoit moyen de faire laisser la grande porte de la maison ouverte, soubz coulleur de quelque maladie (Navarre $1965: 807)$

Il est important d'observer aussi le contexte précédent pour éliminer l'interprétation spatiale. En effet, dans les deux premiers exemples, nous avons le verbe venir, qui indique que les locuteurs sont déjà présents dans les lieux où l'action de l'infinitif se réalise. De même, dans les exemples où l'on comprend qu'une conversation est en cours, l'idée qu'un des locuteurs doive se déplacer pour la continuer est invraisemblable. L'existence d'un échange verbal peut donc constituer un indice à partir duquel on conclut que l'action des verbes déclaratifs est imminente.

En outre, il faut souligner ici que l'action des verbes déclaratifs est non seulement imminente, mais aussi accomplie dès que la personne commence à parler (appeler, raconter...).

Dans l'exemple (3), la périphrase aspectuelle du début de l'action (commencer $a ̀+i n f$.) est insérée à l'intérieur de la construction, ce qui ajoute un élément inchoatif à l'analyse.

Quand l'action de l'infinitif (se coucher) nécessite logiquement un changement de lieu par rapport à l'action précédente, il faudrait analyser la construction comme ayant la valeur spatiale :

7) Quant ses matines out chantees

Li evesques, et definees

A Avrenches, enz el mostier,

Nuit ert encor, va se couchier. (ChrR, v. 2943-2946)

Mais lorsque l'infinitif est lui-même un verbe de mouvement (8), il supprime nécessairement le sémantisme de aller (s'avancer pour courir n'est pas logique) et il s'agit donc d'une périphrase prospective. Nous proposons la même interprétation pour (9) où l'infinitif dénote une activité plus abstraite (se faire couronner, usurper) :

8) Ilz virent leurs ennemis rengiez sur les champs, si leur vont courir sus. (Mél : 235)

9) si fu pris le duc d'Osteriche, comme dit est dessus et sa bataille desconfite; et tantost Loys de Baviere se va faire coronner et usurper les drois de l'Empire en soy appellant roy des Romains semper Augustus en ses lettres ( $\mathrm{GrChr}: 38)$

Dans l'exemple suivant, nous avons des verbes de mouvement dans le contexte précédent (venir, entrer), mais, à la différence des exemples cités plus 
haut, où ces verbes rendent superflu tout mouvement postérieur, ici, on peut facilement imaginer le déplacement à l'intérieur de la pièce. La construction est de valeur spatiale :

10) Et a tant veez cy venir et entrer ses hommes de la garde qui tous ensemble saluerent la compaignie, et puis se vont sarrer en une part contre ung coing de la salle, qui sembloit que tous cent ne tinssent pas la place de quarante. (Rom : 77)

Néanmoins, ni pour (7) ni pour (10), l'interprétation prospective n'est à rejeter. Dans leur article, Bres et Labeau (Bres \& Labeau 2013 : 19) ont analysé un exemple où la construction aller + inf. est analysée comme prospective parce qu'elle est au présent, alors que le contexte est au passé. L'argument pour justifier cette analyse est, à notre avis, pertinent : le verbe aller ne représente plus un mouvement concret, mais un déplacement abstrait. De même, nous ajouterions : pourquoi l'auteur n'a-t-il pas utilisé un temps du passé à la place d'un $v a+i n f$. si le sens spatial était celui qu'il a voulu obtenir ? Nous donnons un exemple similaire de la même œuvre et du même auteur :

11) Quant son mary s'en fut allé et tous ses varletz avec luy, deux pauvres vielles, qui en sa santé l'avoient si longuement gardée, ne se doubtans plus de la perdre, sinon par mort, se vont très bien coucher à leur aise. Et quant elle les ouyt dormyr et ronfler bien hault, se leva toute en chemise et saillist hors de sa chambre (Navarre $1965: 1062)$

Effectivement, pourquoi Marguerite de Navarre a-t-elle utilisé le verbe aller au présent, alors que s'allèrent coucher est une forme courante en français préclassique ? Ou, tout simplement, pourquoi ne pas avoir mis se couchèrent ? Bres et Lebeau (Bres \& Labeau 2013 : 19) expliquent qu'il s'agit d'une « ressource stylistique complémentaire du présent narratif pour dynamiser le récit ». Dans cet exemple, nous avons aussi un indice qui nous suggère que l'action de se coucher découle logiquement des convictions personnelles des « deux pauvres vieilles »: elles ne doutaient pas de perdre leur charge, et, par conséquent, elles sont allées dormir à l'aise. En prenant les choses de ce point de vue-là, on peut tout de suite trouver que, dans (7), l'évêque est allé dormir parce qu'il était encore nuit, ou, dans (10), que Louis de Bavière a usurpé l'Empire et s'est fait couronner une fois son adversaire éliminé.

Donc, dans ces exemples, il est impossible de ne pas remarquer qu'il s'est produit un mouvement qui favorise la lecture spatiale, mais que nous avons aussi la présence d'indices qui suggèrent la lecture prospective. Puisque l'aspect prospectif a directement évolué de l'effet de sens spatial, sans le supplanter pour autant, nous proposons de ne pas séparer les deux lectures coûte que coûte, mais de supposer une ou plusieurs étapes de passage de l'une vers l'autre. 
La construction aller + inf. peut aussi être précédée par un adverbe indiquant une faible distance temporelle entre elle et une action antérieure (maintenant, (et) lors, (et incontinent) après). De cette manière, l'effet d'imminence est renforcé.

12) Quant Lanpars l'ot, grant joie en a.

Vers lui maintenant s'en ala,

Maintenant le va acoler ;

Molt bel sanblant li fait mostrer :

«Sire, molt avés enduré

Et molt travillié et pené [...]» (BelInc : v. 2725-2730)

13) Et lors, a ce mot, Melusigne le va acoler et baisier moult doulcement en disant : "Adieu, mon tres doulz amy, mon bien, mon cuer et toute ma joye [...] » (Mél : 258)

14) $[\ldots]$ car celle qui estoit meneur d'eage, et la devoit honorer et avancier, si l'avoit ainxi reboutée arrieres. Lors va jurer li contes et dist a la contesse sa femme « Par le paterne Dieu, qui est mon serement $[. .] ».(\mathrm{ChrM}: 164)$

15) Et incontinant après va arriver deux cens hommes d'armes tous em point comme pour combatre, et venoient quatre a quatre en moult belle ordonnance et sans bruyt (Rom : 58)

Va accoler de l'exemple (12) est clairement prospectif, parce que le mouvement est déjà exprimé par le verbe s'en aller au passé simple. L'exemple est intéressant parce que l'auteur continue le récit au présent après la périphrase. On observe le même changement de temps verbal dans (14). L'exemple (13) pourrait être interprété comme spatial, bien que nous ayons un indice - a ce mot. Ceci dit, les adverbes d'imminence temporelle pourraient, eux aussi, servir d'incices si le contexte se prête à une interprétation de cause - conséquence. Les deux exemples suivants sont prospectifs parce que le verbe jurer introduit le discours direct (14) et parce que le verbe arriver (15) rend superflu l'idée de mouvement exprimée typiquement par aller.

La lecture spatiale est facilitée si aller + inf. au présent se retrouve dans un contexte au présent de l'indicatif (16 et 17) ou bien s'il y a des compléments circonstanciels de lieu (18) :

16) Une herbe voit an une haie, mout bone por dolor tolir de plaie, et il la va coillir. L'erbe a coillie, si s'an va (Perc : v. 6668-6671)

17) Lors broche le cheval des esperons et estraint l'espee ou poing et va ferir le roy sarrasin sur le heaume par telle vertu qu'il l'embrunche sur le col du destrier (Mél : 180)

18) Al dos le siwent dis mil homes od helmes ;

En l'Archamp vont rei Deramed requere. (ChGui, v. 142-143) 
Lorsque le verbe aller est à l'imparfait, l'interprétation spatiale serait l'interprétation par défaut si le contexte est au passé :

19) Por mialz feire la traïson, L'aloit visiter mout sovant (Cligés : v. 5658-5659)

20) Il ne se tindrent pas atant que il ne feissent vilenie au message le roi ; car endementiers que il aloit une nuit dormir en un solier d'une maison, il errachierent l'entablement qui estoit devant son lit. (GrChr : 78)

Mais si la construction est à l'imparfait dû à une concordance des temps, il s'agit d'aspect prospectif. Dans les trois exemples suivants, les verbes introducteurs du discours indirect sont conter, enquester, panser (penser).

21) Lors li a l'uevre recontee,

Ensi com cil li ot contee

$\mathrm{Ki}$ aloit rescorre s'amie ;

Et il jurent qu'il n'ira mie (Âtre : v. 4519-4522)

22) Or est temps que je vous parle de Guyon et du maistre de Rodes, qui avoient enquesté aux Sarrasins que ilz tenoient prisonniers, ou la grosse flote aloit prendre terre, et ceulx leur dirent : En Chippre. (Mél : 130)

23) Si panserent que il aloient assaillir messire Nicole le Maure et sa compaignie (ChrM : 369)

En ce qui concerne l'interprétation futurale, nous n'avons trouvé aucun exemple dans notre corpus. Ceci peut être dû non seulement au fait que le futur périphrastique ne se manifeste explicitement que vers l'époque classique, mais aussi au fait que la première personne du singulier est rarement utilisée dans les anciens textes narratifs et, par conséquent, nous ne pouvons pas vérifier l'argument du DMF ${ }^{1}$. Néanmoins, nous citerons ici trois exemples du XvI ${ }^{e}$ siècle avec la première personne du singulier.

24) «[...] je vous apprendray à confesser que la nature des femmes et des hommes est de soy incline à tout vice, $[\ldots]$ et pour vous abbatre l'audace que vous prenez, quant on en dit à vostre honneur, je vous en vais montrer un exemple qui est très veritable. » (Navarre 1965 : 946)

25) Que deviendrai-je moi pauvrette ?

Resterai-je en ce monde ici, Voyant mon frère en tel souci?

Mon esprit fuira comme vent : Z̆

Mais je vais courir au-devant, Je veux l'infortune savoir. (Jodelle $1968: 55$ )

\footnotetext{
${ }^{1} c f$. chapitre 2.3. sur le futur périphrastique.
} 
26) Maintenant en doute je suis

S'on n'auroit point changé nostre huis.

J'y va voir pour le reconoistre.

Tout y est comme il souloit estre. (Baïf 1979 : 93)

Les exemples sont prospectifs, puisque les locuteurs annoncent leur intention de réaliser une action dans les plus brefs délais. On dit qu'il s'agit de l'implication personnelle du sujet (Stanojević 2014 : 192). Dans (25), le verbe courir empêche en plus la lecture spatiale.

Si l'on essayait de vérifier la possibilité d'interpértation futurale en remplaçant les paraphrases par des FS, on verrait que c'est impossible.

\section{Conclusion}

Pour peu que le chercheur s'intéresse à l'origine de la construction communément appelée " futur proche » ou « futur périphrastique », il découvre rapidement que, dans une certaine époque du développement de la langue française, cette construction n'exprimait pas une action future, mais qu'il s'agissait d'une périphrase aspectuelle. Si l'on remonte encore plus dans le temps, la construction perd aussi son épithète "périphrastique » et c'est seulement à l'aide du sémantisme du verbe aller que l'action du verbe à l'infinitif peut être considérée comme appartenant au procès suivant. Au cours de notre analyse des exemples concrets, nous avons réussi à confirmer que non seulement les affirmations de Vet et de Bres sont valables, mais qu'il y a aussi plusieurs facteurs à observer afin de déterminer quelle interprétation est à adopter.

En se penchant sur le contexte de chaque phrase, nous avons repéré d'abord que le sémantisme des verbes y joue un rôle dominant, qu'il s'agisse de l'infinitif faisant partie de la construction ou des autres verbes du contexte. Puis, l'emploi de certains temps verbaux (dans le contexte et à l'intérieur de la construction), l'existence de certains adverbes (temporels), ainsi que les indices de nature pragmatique apportent des informations cruciales. Néanmoins, il semble que l'analyse de la construction aller + infinitif ne puisse être strictement réduite aux trois niveaux d'interprétation que nous proposons. En effet, il y a un nombre d'exemples qui peuvent être interprétés comme spatiaux et comme prospectifs.

À défaut d'exemples explicites de futur périphrastique, nous n'avons pu déterminer son degré d'interaction avec le sens aspectuel, qui existe en français moderne. 


\section{Sources}

Âtre - Anonyme, Âtre périlleux. 〈http://catalog.bfm-corpus.org/atrper〉. 01/11/2018.

ChGui - Anonyme, Chanson de Guillaume. 〈http://catalog.bfm-corpus.org/guill1〉. $01 / 11 / 2018$.

ChrM - Anonyme, Chronique de Morée. 〈http://catalog.bfm-corpus.org/moree〉. 01/11/2018.

Floire - Anonyme, Floire et Blancheflor. 〈http://catalog.bfm-corpus.org/floire jl〉. 01/11/2018.

Grchr - Anonyme, Grandes chroniques de France, tome IX, Charles IV Le Bel. 〈http:// catalog.bfm-corpus.org/grchron9>. 01/11/2018.

Rom - Anonyme, Roman de Jean de Paris. 〈http://catalog.bfm-corpus.org/jehpar〉. 01/11/2018.

Gen - Anonyme, Vie de sainte Geneviève (version V). 〈http://catalog.bfm-corpus.org/ SGenPr5>. 01/11/2018.

Mél - Arras, J. d', Mélusine. 〈http://catalog.bfm-corpus.org/melusine〉. 01/11/2018.

Baïf 1979 : J.-A. de Baïf, Le Brave, Genève : Droz.

BelInc - Beaujeu, R. de, Bel Inconnu. 〈http://catalog.bfm-corpus.org/belinc〉. 01/11/2018. Jouv-Bueil, J. de, Le Jouvencel. 〈http://catalog.bfm-corpus.org/jouvencel2〉. 01/11/2018. Jodelle 1968 : É. Jodelle, Oeuvres complètes, t.2, Paris : Gallimard.

Navarre 1965 : M. de Navarre, Conteurs français du $16^{e}$ siècle, Paris : Gallimard.

ChrR - Saint Pair, G. de, Chronique rimée du Mont Saint Michel. «http://catalog.bfmcorpus.org/ChronSMichelBo〉. 01/11/2018.

CJouv - Tringant, G, Commentaires du Jouvencel. 〈http://catalog.bfm-corpus.org/tringant>. 01/11/2018.

Cligés - Troyes, C. de, Cligés. 〈http://catalog.bfm-corpus.org/CligesKu〉. 01/11/2018.

Érec - Troyes, C. de, Érec et Énide. 〈http://catalog.bfm-corpus.org/ErecKu〉. 01/11/2018.

Perc - Troyes, C. de, Conte du Graal (Perceval). 〈http://catalog.bfm-corpus.org/PercevalKu>. 01/11/2018.

\section{Références bibliographiques}

BFM 2016 : Base de Français Médiéval, Lyon : ENS de Lyon, Laboratoire IHRIM. 〈http://www.txm.bfm-corpus.org〉. 01/11/2018.

Barbazan 2010 : M. Barbazan, « Comment choisir le meilleur de futurs ? contraintes et libertés d'usage pour le Futur Simple et le Futur Composé (aller + infinitif) », L'Information grammaticale, $\mathrm{n}^{\circ} 127,3-9$.

Bres \& Labeau 2013 : J. Bres \& E. Labeau, « Aller et venir des verbes de déplacement aux auxiliaires aspectuels-temporels-modaux », Langue française, vol. 179, $\mathrm{n}^{\circ} 3$, $13-28$.

DMF 2015 : Dictionnaire du moyen français, ATILF - CNRS \& Université de Lorraine. (http://www.atilf.fr/dmf〉. 01/11/2018.

Frantext 1998-2018 : ATILF, Nancy. 〈http://www.frantext.fr〉.

Helland 1995 : H.P. Helland, « Futur simple et futur périphrastique : du sens aux em- 
plois », Revue Romane, vol. 30, n 1, 3-26.

Revaz 2002 : F. Revaz, « Le présent et le futur « historiques » : Des intrus parmi les temps du passé ? », Le français aujourd'hui, n 139, 87-96.

Stanojević 2014 : V. Stanojević, « Quelques réflexions sur le futur périphrastique et son rapport au futur simple », in M. Vinaver-Ković et V. Stanojević (dir.), Les Études françaises aujourd'hui (2014), Belgrade : Faculté de Philologie de l'Université de Belgrade, 181-198.

Stavinohova 1977 : Z. Stavinohova, «Le futur « proche » et le futur simple dans la langue littéraire contemporaine », Études romanes de Brno, vol. 9, 115-126.

Vet 2008 : C. Vet, «Six traits sémantiques suffisent à décrire tous les temps du français », in : M. Birkelund, M.-B. Mosegaard Hansen et C. Norén (dir.), L'énonciation dans tous ses états :Mélanges offerts à Henning Nølke à l'occasion de ses soixante ans, Berne : Peter Lang, 451-471.

Vet 2011 : C. Vet, «L'aspect grammatical en français », in F. Neveu, P. Blumenthal et N. Le Querler (dir.), Au commencement était le verbe. Syntaxe, sémantique, cognition, Berne : Peter Lang, 443-465.

\section{Милена Анђелић}

\section{Развој значења перифразе aller + инфинитив}

У овом раду покушаћемо да провјеримо оправданост Ветових $(2008,2010)$ и Бресових (2013) аргумената о развоју значења конструкције aller + инфинитив. Како бисмо провјерили њихову претпоставку да је у старијим облицима француског језика постојала само аспектуална интерпретација ове конструкције, анализираћемо примјере из текстова од XII до XVI вијека и одредићемо да ли се они могу сврстати под једну од три категорије: 1) интерпретација кретања како би се остварила одређена радња; 2) проспективни аспекат; 3) перифрастични футур. У предкласичном преиоду развоја француског језика требало би да се примјери слажу само са интерпретацијама кретања и проспективности, али не и футуралности, јер ће се перифрастични футур појавити тек око XVII вијека.

Кључне ријечи: проспективни аспекат, припремна фаза, перифрастични футур, граматикализација, глаголско вријеме. 